\title{
The transmembrane serine protease (TMPRSS3) mutated in deafness DFNB8/10 activates the epithelial sodium channel (ENaC) in vitro
}

\author{
Michel Guipponi ${ }^{1, \dagger} \ddagger$, Grégoire Vuagniaux ${ }^{2, \dagger}$, Marie Wattenhofer ${ }^{1,3}$, Kazunori Shibuya ${ }^{4}$, \\ Maria Vazquez ${ }^{5}$, Loretta Dougherty ${ }^{6}$, Nathalie Scamuffa ${ }^{1}$, Elizabeth Guida ${ }^{7}$, \\ Michiyo Okui ${ }^{4}$, Colette Rossier ${ }^{1}$, Manuela Hancock ${ }^{6}$, Karine Buchet ${ }^{1}$, Alexandre Reymond ${ }^{1}$, \\ Edith Hummler ${ }^{2}$, Phillip L. Marzella ${ }^{7}$, Jun Kudoh ${ }^{4}$, Nobuyoshi Shimizu ${ }^{4}$, Hamish S. Scott ${ }^{6}$, \\ Stylianos E. Antonarakis ${ }^{1, *}$ and Bernard C. Rossier ${ }^{2}$
}

${ }^{1}$ Division of Medical Genetics, University of Geneva Medical School, and Geneva University Hospitals, Switzerland, ${ }^{2}$ Institut de Pharmacologie et de Toxicologie, Université de Lausanne, Switzerland, ${ }^{3}$ Graduate Program of Molecular and Cellular Biology, University of Geneva Medical School, Geneva, Switzerland, ${ }^{4}$ Department of Molecular Biology, Keio University School of Medecine, 35 Shinanomachi, Shinjuku-ku, Tokyo 160-8582, Japan, ${ }^{5}$ Department of Morphology, University of Geneva Medical School, Geneva, Switzerland, ${ }^{6}$ Genetics and Bioinformatics Division, The Walter and Eliza Hall Institute of Medical Research, Royal Parade, Parkville, P.O. Royal Melbourne Hospital, Victoria 3050, Australia and ${ }^{7}$ Department of Otolaryngology, The University of Melbourne, Royal Victorian Eye and Ear Hospital, Victoria 3050, Australia

Received June 6, 2002; Revised and Accepted August 26, 2002 DDBJ/EMBL/GenBank accession numbers AJ429216 and AJ300738

TMPRSS3 encodes a transmembrane serine protease that contains both LDLRA and SRCR domains and is mutated in non-syndromic autosomal recessive deafness (DFNB8/10). To study its function, we cloned the mouse ortholog which maps to Mmu17, which is structurally similar to the human gene and encodes a polypeptide with $\mathbf{8 8 \%}$ identity to the human protein. RT-PCR and RNA in situ hybridization on rat and mouse cochlea revealed that Tmprss 3 is expressed in the spiral ganglion, the cells supporting the organ of Corti and the stria vascularis. RT-PCR on mouse tissues showed expression in the thymus, stomach, testis and E19 embryos. Transient expression of wild-type or tagged TMPRSS3 protein showed a primary localization in the endoplasmic reticulum. The epithelial amiloride-sensitive sodium channel (ENaC), which is expressed in many sodium-reabsorbing tissues including the inner ear and is regulated by membrane-bound channel activating serine proteases (CAPs), is a potential substrate of TMPRSS3. In the Xenopus oocyte expression system, proteolytic processing of TMPRSS3 was associated with increased ENaC mediated currents. In contrast, 6 TMPRSS3 mutants (D103G, R109W, C194F, W251C, P404L, C407R) causing deafness and a mutant in the catalytic triad of TMPRSS3 (S401A), failed to undergo proteolytic cleavage and activate ENaC. These data indicate that important signaling pathways in the inner ear are controlled by proteolytic cleavage and suggest: (i) the existence of an auto-catalytic processing by which TMPRSS3 would become active, and (ii) that ENaC could be a substrate of TMPRSS3 in the inner ear.

\section{INTRODUCTION}

Hearing loss is the most common sensory defect in humans, affecting about one in 1000 children (1). About $60 \%$ of all cases of sensorineural deafness are of genetic origin (2) and non-syndromic autosomal recessive deafness accounts for $\sim 70 \%$ of genetic hearing loss cases (2). Thirty-three loci that cause autosomal recessive non-syndromic hearing loss

\footnotetext{
*To whom correspondence should be addressed at: Division of Medical Genetics, Centre Médical Universitaire, 1 rue Michel-Servet, CH-1211 Geneva 4, Switzerland. Tel: +41 227025708; Fax: +41 227025706; Email: stylianos.antonarakis@medecine.unige.ch

Present address:

Genetics and Bioinformatics Division, The Walter and Eliza Hall Institute of Medical Research, Royal Parade, Parkville,

P.O. Royal Melbourne Hospital, Victoria 3050, Australia.

\$The authors wish it to be known that, in their opinion, the first two authors should be regarded as joint First Authors.
} 
have been identified and seventeen of these genes have been isolated (http://dnalab-www.uia.ac.be./dnalab/hhh/index.html). Mutations in the TMPRSS3 gene, which encodes a transmembrane serine protease, were identified in both familial and sporadic cases of non-syndromic sensorineural recessive deafness, DFNB8/10 (3-6). TMPRSS3 (for transmembrane protease, serine 3) is a type II transmembrane serine protease (TTSP) and like the other members of this family, it contains a short N-terminal tail, a transmembrane (TM) domain and a large C-terminal segment characterized by: (i) a stem region containing LDLRA and SRCR domains; (ii) a potential proteolytic activation cleavage site between R216 and I217; and (iii) a serine protease domain containing the catalytic triad signature (H257, D304 and S401) (Fig. 1B). This structure suggests that TMPRSS3 could be active as a membrane-bound enzyme (7).

Channel activating proteases (CAPs) are membrane-bound serine proteases that are able to activate $\mathrm{ENaC}$ in vitro $(8,9)$. Recently, three CAPs have been identified in a mouse kidney cell line (mCAP1, mCAP2, mCAP3) (10). Interestingly, like TMPRSS3, mCAP2 is a type II transmembrane serine protease incorporating LDLRA and SRCR domains. MCAP2 is the mouse ortholog of hTMPRSS4. Recently, it has been suggested that $\mathrm{ENaC}$ plays a role in the establishment and maintenance of the remarkably low sodium concentration of the endolymph $(11,12)$. This observation raises the possibility that $\mathrm{ENaC}$ could be a substrate of TMPRSS3 in the inner ear.

To study the function of TMPRSS3 in the inner ear, we isolated the mouse Tmprss 3 and characterized its expression profile and the intracellular localization of the human TMPRSS3 protein. We show that Tmprss 3 co-localizes with $\mathrm{ENaC}$ in some inner ear tissues and that, unlike wild-type TMPRSS3, TMPRSS3 mutants causing deafness fail to undergo proteolytic processing and to activate $\mathrm{ENaC}$ in vitro.

\section{RESULTS}

\section{Isolation of the mouse Tmprss 3 cDNA and gene}

Using the methods described later, we constructed a $2752 \mathrm{bp}$ mouse Tmprss 3 cDNA encoding a putative protein of 453 amino acids that showed $88 \%$ identity to human TMPRSS3 (Fig. 1B) (3). Similar to TMPRSS3, Tmprss3 is divided into 12 coding exons over $18 \mathrm{~kb}$ of genomic sequence, but there was no experimental evidence for the existence of a $5^{\prime} \mathrm{UTR}$ exon in the mouse gene (Fig. 1A). No alternative mouse transcripts were identified.

\section{Tmprss3 mRNA expression}

RT-PCR was performed to study the expression profile of Tmprss3. Significant expression was found in thymus, stomach, testis, E19 embryos, stria vascularis, modiolus, organ of Corti and cultured spiral ganglion (Figs. 2A and B). No amplification from genomic DNA was observed.

To confirm these data, RNA in situ hybridization was performed on mouse embryos and inner ear sections. Tmprss 3 expression was not detected in the otocyst of mouse embryos from E8.5 to E10.5 d.p.c. (data not shown). Further analyses were performed on mouse postnatal day 5 when the inner ear development is almost complete. Tmprss 3 expression was detected in the spiral ganglion and in the entire epithelium that supports cells of the organ of Corti but was not observed in sensory hair cells. Weak expression was also found in the stria vascularis (Fig. 3).

\section{Intracellular localization of human TMPRSS3 protein}

The intracellular localization of the TMPRSS3 protein was investigated by using wild-type and $\mathrm{N}$ - or C-terminal tagged TMPRSS3 proteins. All TMPRSS3 proteins tested, showed cytoplasmic localization in HeLa, Cos-7, U2OS and MDCK cells (Fig. 4A-D, G and data not shown). Co-localization experiments showed that the TMPRSS3 protein is primarily localized in the endoplasmic reticulum membranes where it may be anchored by its transmembrane domain (Fig. 4F and I and data not shown).

\section{Expression analysis of the $\alpha$-, $\beta$ - and $\gamma$-ENaC subunits in the rat inner ear}

The $\mathrm{ENaC}$ channel is expressed in the inner ear and is known to be regulated by serine protease activity. These features make it a candidate substrate for TMPRSS3. To investigate this possibility, the co-expression of Tmprss 3 and the three ENaC subunits was analysed by RT-PCR on a panel of rat inner ear tissues. Similar to Tmprss $3, \alpha$ - and $\gamma$-subunits were detected in all the samples tested. The $\beta$-subunit was detected in all samples tested except the organ of Corti and the spiral ganglion, even after nested PCR (Fig. 2B).

\section{Functional and biochemical analysis of TMPRSS3 in Xenopus oocytes}

The six TMPRSS3 missense mutations associated deafness can be divided into three main groups: (i) LDLRA domain mutants (D103G, R109W); (ii) an SRCR domain mutant (C194F); and (iii) serine protease domain mutants (W251C, P404L, C407R) (4-6), (Fig. 5A). Amino acid sequence comparisons of a portion of the LDLRA, SRCR and serine protease domains of the human and mouse TMPRSS3 with few selected such domains of other proteins are available at http://medgen.unige.ch/ public_1.html

Co-expression of the human WT-TMPRSS3 cRNA with the rat $\mathrm{ENaC}$ subunits $(\mathrm{rENaC})$ led to an increase in $\mathrm{ENaC}$ mediated current $\left(\mathrm{I}_{\mathrm{Na}}\right)(P<0.01)$ compared to oocytes injected only with $\mathrm{rENaC}$ (Fig. 5B, lane 2 versus lane 1). Oocytes injected only with water or TMPRSS3 did not generate measurable current (data not shown). The LDLRA domain mutants (D103G, R109W) retained a residual but significant $(P<0.05)$ activity (lanes 3 and 4 versus lane 2$)$. The SRCR domain mutant (C194F) was totally inactive (lane 5 versus lane 2). The catalytic domain mutants were either totally inactive (W251C, P404L) (lanes 6 and 7 versus lane 2) or partially active (C407R), leading to a small but significant $(P<0.05) \mathrm{ENaC}$ activation (lane 8 versus lane 2$)$. As a negative control, the S401A catalytic triad mutant was totally inactive (lane 9 versus lane 2). Expression of the pro domain (M1-S215) of TMPRSS3 (pro domain, pro) decreased baseline activity $(P<0.01$, lane 10 versus lane 1$)$ whereas the serine protease domain (M-C204-T454) of TMPRSS3 (SP domain, $\mathrm{SP}$ ) was inactive (lane 11 versus lane 2). As shown in 

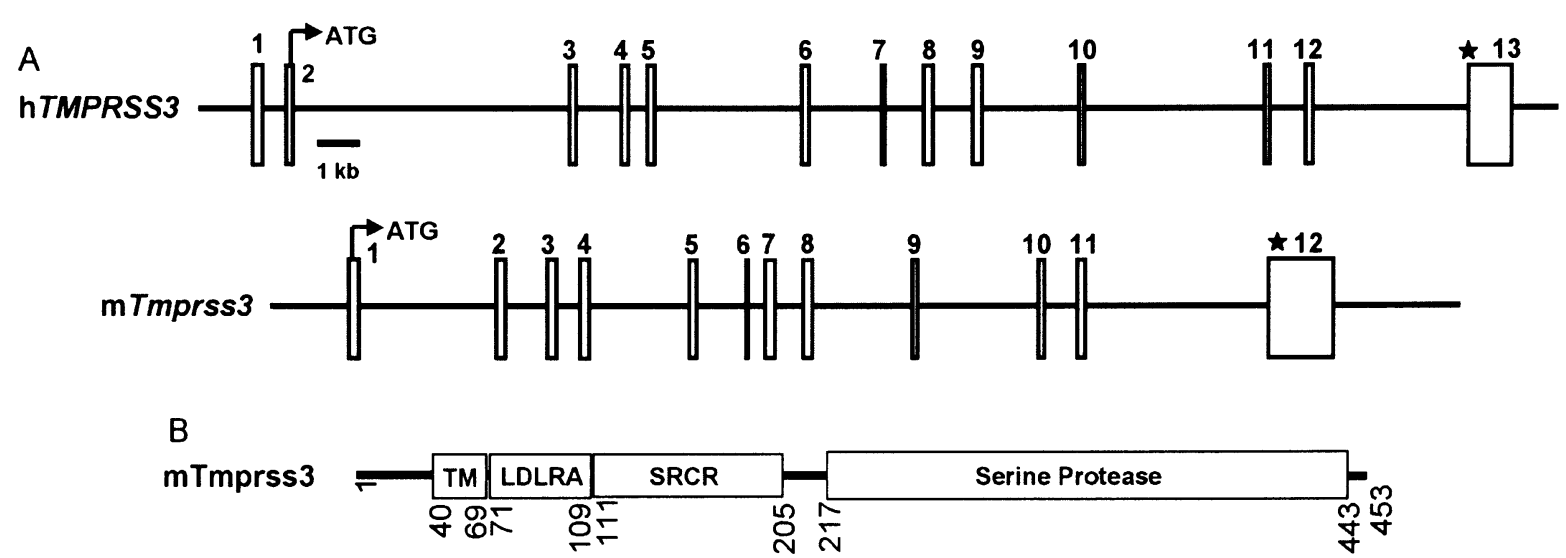

Figure 1. (A) Schematic representation of the human and mouse TMPRSS3 genomic structure. Exons are represented as boxes numbered on top, with start and stop/codons indicated. Introns are shown as thin lines. (B) Schematic representation of the mouse Tmprss3 protein showing the TM, LDLRA, SRCR and serine protease domains and their position in the 453 amino acid polypeptide.

Figure 5C, trypsin was able to activate $\mathrm{ENaC}$ significantly in the absence of TMPRSS3 (lane 1). When ENaC was co-expressed with WT-TMPRSS3, however, subsequent exposure to trypsin had only a small additional effect (lane 2) indicating a non-additive, common signalling pathway between trypsin and TMPRSS3. By contrast, when ENaC was co-expressed with TMPRSS3 mutants (lanes 3-9), pro (lane 10) or SP domains (lane 11), sodium currents were significantly enhanced by subsequent perfusion with trypsin.

When expressed in Xenopus oocytes, WT-TMPRSS3 was detected as three protein species on a western blot analysis: a predominant doublet of 50 and $54 \mathrm{kDa}$ and a faint $30 \mathrm{kDa}$ fragment suggesting that a significant fraction of TMPRSS3 was proteolytically processed (Fig. 5D). The faint $30 \mathrm{kDa}$ fragment migrated at the same position as the catalytic serine protease domain (SP domain, lane 11) consistent with an auto-proteolytic cleavage at the activation site between R216 and I217, as occurs on other TTSPs (7). The $25 \mathrm{kDa}$ non-catalytic subunit of TMPRSS3 (pro domain, lane 10) could not be detected for WT-TMPRSS3, suggesting additional proteolytic cleavage(s). The unprocessed isoform of TMPRSS3 was detected for all TMPRSS3 deafness mutants (lanes 3-8) and the S401A mutant (lane 9) indicating that they were all well expressed but not cleaved in the Xenopus oocyte expression system. These results indicate that the LDLRA and SRCR domains and the serine protease domain are all implicated in the $\mathrm{ENaC}$ channel activation by TMPRSS3 and in the processing of TMPRSS3.

\section{DISCUSSION}

After our identification of TMPRSS3 as the gene responsible for DFNB8/10 (3), we identified additional mutations in TMPRSS3 (4-6) and pursued a variety of approaches to investigate the role of TMPRSS3 in the hearing process. In this paper, we report the isolation of the mouse ortholog and subsequent expression studies. We also report that TMPRSS3 which may localize in the endoplasmic reticulum membranes, undergoes cleavage and activates ENaC whereas the TMPRSS3 mutant proteins causing deafness are all unprocessed and inactive. We also demonstrate that $\mathrm{ENaC}$ is co-expressed with Tmprss3 in the inner ear.

The specific ion composition of the endolymph, characterized by high potassium and low sodium concentrations, is essential for the proper function of the sensory hair cells. Genes important for potassium recycling are mutated in different forms of syndromic and non-syndromic deafness $(13,14)$. In contrast, little is known about the transport systems and sites involved in sodium homeostasis. The $\mathrm{ENaC}$ channel is a highly selective sodium channel that is expressed in many sodium-reabsorbing tissues and is a good candidate for sodium reabsorption from the endolymph. Indeed, the three $\mathrm{ENaC}$ subunits mRNAs have been detected in rat inner ear. Their expression levels were found to increase from postnatal day 2 reaching the highest levels of expression at postnatal day 12 when the concentration of sodium in endolymph reaches adult levels $(11,15)$. These data suggest that $\mathrm{ENaC}$ may be involved in the regulation of sodium concentration in the endolymph $(11,12)$.

Using a Xenopus oocyte expression system, we showed that TMPRSS3 is proteolytically cleaved and significantly activates ENaC. In addition, we showed that TMPRSS3 missense mutations causing deafness, that lie in all identified domains except the TM, and a catalytic triad S401A mutant, all fail to undergo proteolytic cleavage and to activate ENaC. The mechanism of ENaC activation by TMPRSS3 remains elusive and could be direct or indirect. TMPRSS3 anchored in the endoplasmic reticulum membranes may play a role in the processing of $\mathrm{ENaC}$. $\mathrm{ENaC}$ is assembled and acquires core glycosylation in the endoplasmic reticulum; this subunit assembly occurs early in channel biosynthesis and is an important determinant of ENaC maturation (16).

All the TMPRSS3 pathogenic missense mutations described so far, D103G, R109W, C194F, W251C, P404L and C407R (Figs. 5A), affect highly conserved residues except $\mathrm{R} 109 \mathrm{~W}$ in the LDLRA domain and C407R in the serine protease domain (4-6). Based on our functional screening of TMPRSS3 mutants for their activation of $\mathrm{ENaC}$ in a Xenopus oocyte expression system, the mutations can be classified into three groups: (i) LDLRA domain mutants D103G and R109W which are partially active; (ii) an SRCR domain mutant, C194F, which 

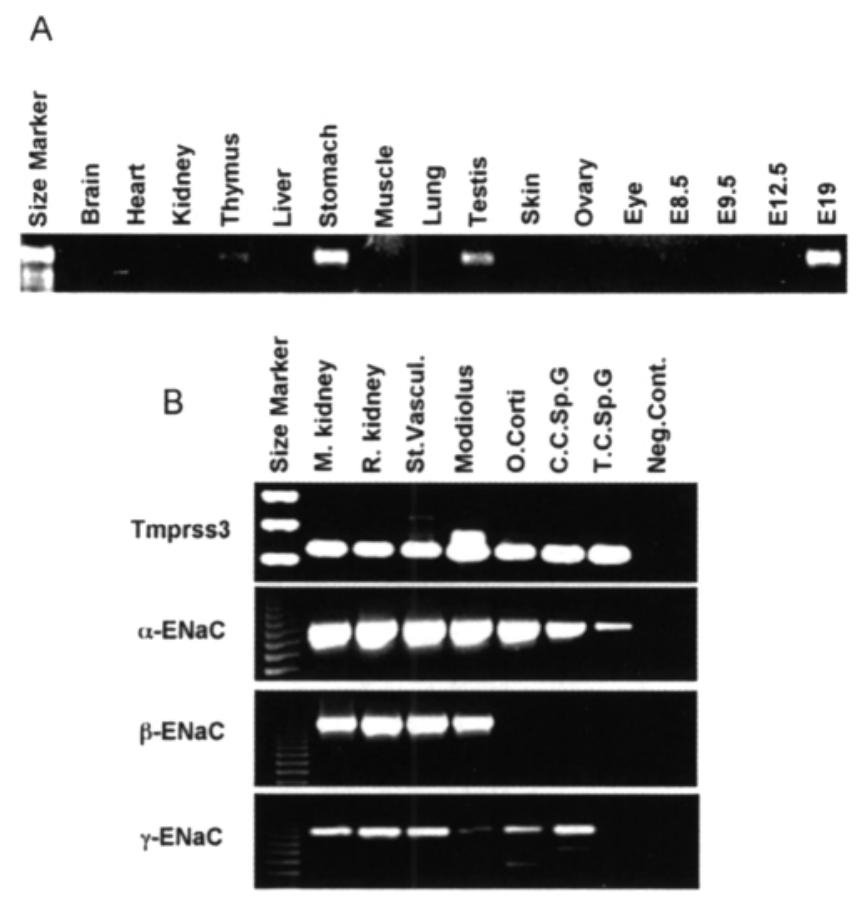

Figure 2. (A) RT-PCR analysis of mouse Tmprss 3 expression. (B) RT-PCR analysis of the expression of Tmprss3, ENaC $\alpha$-, $\beta$ - and $\gamma$-subunits in mouse (M.Kid) and rat kidney (R.Kid), stria vacsularis (St.Vascul.), modiolus, organ of Corti (O.Corti), control cultured spiral ganglion (C.C.Sp.G) and BDNF-treated cultured spiral ganglion (T.C.Sp.G). Nested PCR amplification is presented for Tmprss 3 and $\alpha-E N a C$ subunit. First PCR amplification is presented for $\beta$ - and $\gamma$-ENaC subunits.

changes a highly conserved residue and is totally inactive; and (iii) two subgroups of serine protease domain mutants, the first containing totally inactive mutants in which highly conserved residues are affected, W251C and $\mathrm{P} 404 \mathrm{~L}$, while the second contains a partially active mutant, C407R, in which a conserved amino acid is substituted (Fig. 5A-D).

In order to investigate whether $\mathrm{ENaC}$ could be a substrate of TMPRSS3 in the inner ear, their respective expression profiles were determined and compared. In 2001, Grunder et al. (11) reported expression of the three ENaC subunits in the cochlea of 12-day-old rats by RNA in situ hybridization. $\alpha$-, $\beta$ - and $\gamma$ $\mathrm{ENaC}$ subunits were identified in the supporting cells of the organ of Corti, more specifically Deiters' and Claudius' cells. Only a faint staining in the Claudius' cells was observed for the $\beta$ subunit. Expression of the three subunits was also detected in the spiral ganglion. However, none of the subunit was observed in the sensory hair cells and no significant expression of any of the three subunits was detected in the stria vascularis. In agreement with these data, our RT-PCR study on inner ear tissues of 5-day-old rats showed expression of the $\alpha$ - and $\gamma$ $\mathrm{ENaC}$ subunits in the organ of Corti and in the spiral ganglion and $\alpha, \beta$ and $\gamma$ subunits in the stria vascularis. While $\beta$ subunit expression was observed in the organ of Corti and in the spiral ganglion by in situ hybridization (11), our RT-PCR experiment did show expression of the $\beta$ subunit in these tissues. This apparent discrepancy could be explained by the two different stages of development studied, 12-day-old versus 5-day-old rats, and/or by the fact that in situ hybridization is more efficient at detecting expression in very few cells. Indeed, Grunder et al. (11) found consistently that the $\beta$ subunit was only present in Claudius' cells and not in the Deiters' cells. In any case, from our data and those of Grunder et al. (11), one can propose that ENaC and TMPRSS3 co-localize in the spiral ganglion, in the stria vascularis and in the supporting cells of the organ of Corti. Interestingly, the stria vascularis and the organ of Corti are two cochlear tissues that border the endolymph and are implicated in sodium reabsorption (17-21).

$\mathrm{ENaC}$ is a heterotetrameric channel, $\alpha 2 \beta \gamma$ but channel activity can also be observed in the presence of heterodimers $\alpha 2 \beta 2$ or $\alpha 2 \gamma 2(22,23)$. As far as the response to trypsin in the Xenopus oocyte expression system is concerned, we have found that trypsin can activate $\alpha 2 \beta \gamma$ channels in a similar manner to the CAP proteins. Interestingly, $\alpha 2 \beta 2$ complexes are not sensitive to trypsin or CAPs, whereas $\alpha 2 \gamma 2$ complexes are highly sensitive to trypsin or CAPs. If the oocyte experiments are valid models for what happens in intact cells in vivo, one could speculate that in the cochlea, a relatively small amount of $\alpha 2 \beta \gamma$ channels can be made. If the $\beta$ subunit mRNA is limiting, the predominant form would be an $\alpha 2 \gamma 2$ channel which keeps its trypsin/CAP sensitivity. Since both $\alpha 2, \beta, \gamma$ and $\alpha 2, \gamma 2$ can be activated by CAPs (B.C. Rossier, unpublished observations), it is reasonable to conclude that inner ear cells may express $\mathrm{ENaC}$ with distinct subunit composition and thus different sodium transport capacity but yet controlled by CAPs.

Little is known about the role of $\mathrm{ENaC}$ in the hearing process. In mice, the concentrations of all the ions in endolymph reach adult levels at day 7 after birth. In the earliest postnatal days, the endolymphatic sodium concentration is significantly higher than that in adult mice. $\alpha-\mathrm{ENaC}(-/-)$, $\beta$-ENaC $(-/-)$ and $\gamma$-ENaC $(-/-)$ mice die within $40 \mathrm{~h}$ of birth (24-26). Therefore, they are not convenient models to study the role of $\mathrm{ENaC}$ in the development of the inner ear sodium homeostasis which occurs during the first week of life. To rescue cochlear hair cells from perinatal death of $\alpha-\mathrm{ENaC}$ $(-/-)$, organotypic cultures were performed. Stimulation of the outer hair cells in culture produced transducer currents that were not different from those observed in controls, suggesting a normal vestibular function and mechano-electrical transduction (27). The human $\mathrm{ENaC}$ genes have been found to be mutated in two distinct human diseases, Liddle's syndrome (OMIM\#177200) and autosomal recessive pseudohypoaldosteronism type 1 (PHA-1) (OMIM\#264350). In both conditions, the $\mathrm{ENaC}$ activity is dysregulated leading to disorders of systemic blood pressure (28). Until now, no severe hearing impairment has been reported in those patients but evaluation of less severe or selective impairment is currently underway in PHA-1 patients. ENaC may be only one of several sodium transporters involved in maintaining such an unusually low sodium concentration and some redundancy would be highly desirable in this context. In addition it is unlikely that $\mathrm{ENaC}$ is the only substrate for TMPRSS3 in vivo.

Besides the ion transporters discussed above, other substrates of TMPRSS3 in the inner ear could be neurotrophins. Studies over the past decade have provided evidence that brain-derived neurotrophic factor (BDNF) and neurotrophin 3 (NT-3) and their associated high-affinity tyrosine kinase receptors (TrkB and TrkC, respectively) are necessary for the normal development of the afferent innervation of the inner ear (29). The biological 


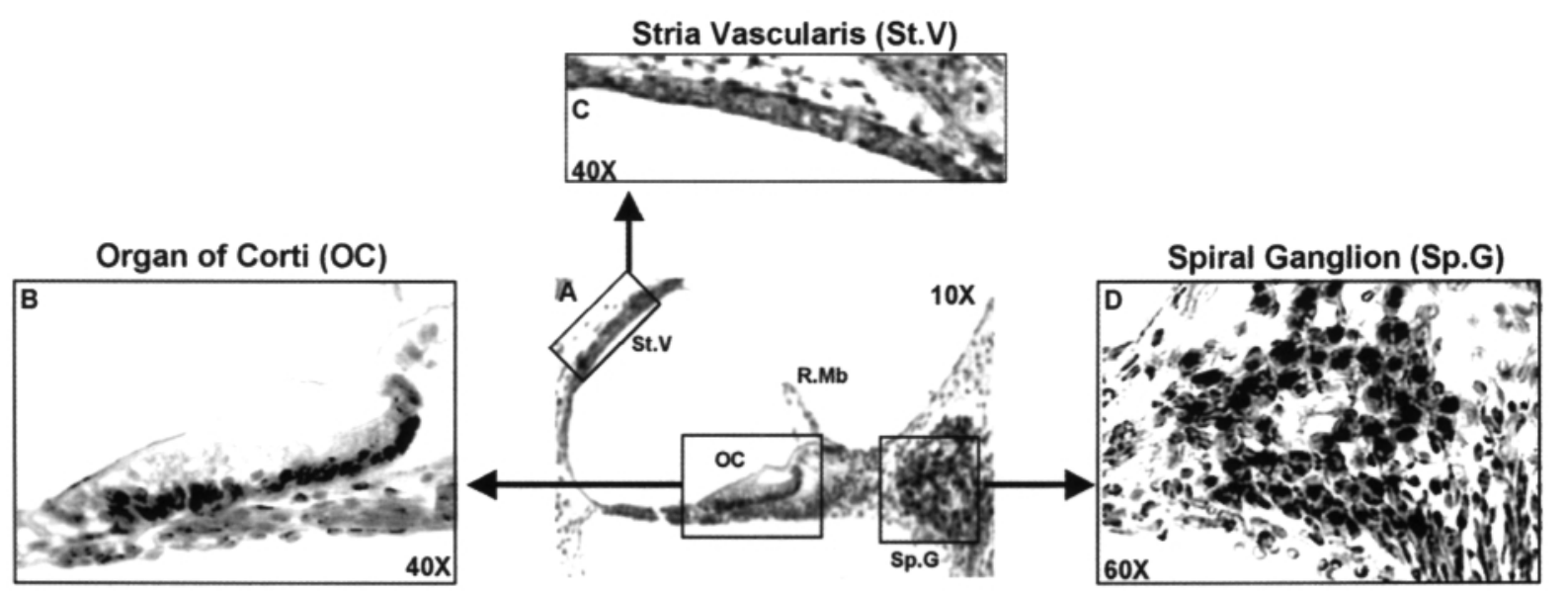

Cochlear duct, Tmprss 3 anti-sense

Figure 3. RNA in situ hybridization of mouse Tmprss 3 in cochlea of postnatal day-5-old mice. Tmprss 3 mRNA was detected in the supporting cells of the organ of Corti (panel A, magnification $10 \times$ and panel B, magnification $40 \times$ ), in the stria vascularis (panel A, magnification $10 \times$ and panel $\mathbf{C}$, magnification $40 \times$ ) and in the spiral ganglion (panel A, magnification 10× and panel D, magnification $60 \times$ ). St.V, stria vascularis; OC, organ of Corti; Sp.G, spiral ganglion; R.Mb, Reissner's membrane.

action of neurotrophins is regulated by proteolytic cleavage of a proform (proneurotrophins) to release the mature active neurotrophins. The serine protease plasmin, which belongs to the same protease family as TMPRSS3, has been shown to cleave the proform of BDNF (30). Neurotrophins, BDNF, NT-3 and their associated receptors, TrkB and TrkC, are all expressed in the organ of Corti and/or spiral ganglion (31-33). These data suggest that TMPRSS3 might also be involved in the processing of proneurotrophins and thus in the development and survival of inner ear innervation.

The results reported in this study contribute to our understanding of the role of TMPRSS3 in the inner ear and the possible etiology of DFNB8/10. We investigated the ENaC sodium channel as a potential substrate of TMPRSS3 in the inner ear focusing on the sodium homeostasis of the endolymph. Another hypothesis, not tested here, is that TMPRSS3 is involved in processing proneurotrophins and thus in the development and survival of the cochlear neurons. The generation of a mouse with targeted disruption of Tmprss 3 will provide a suitable model to investigate these and other hypotheses concerning the molecular mechanisms causing deafness in DFNB8/10.

\section{MATERIALS AND METHODS}

\section{Isolation of the mouse Tmprss 3 cDNA and gene}

A $220 \mathrm{~kb}$ mouse BAC clone, 556K2 (CITB-CJ7-B BAC library; Research Genetics, Paisley, UK), that spans the region of mouse chromosome 17q syntenic to the human TMPRSS3 locus on human chromosome $21 \mathrm{q} 22.3$ has previously been reported (34). It contains the Trefoil 3 gene, which in the human genome, is $\sim 60 \mathrm{~kb}$ centromeric to TMPRSS3. A $1 \mathrm{~kb}$ fragment of the mouse Tmprss 3 was PCR-amplified from BAC 556K2 using degenerate primers $\left(5^{\prime}\right.$-TGYTGGAYNWSNGGNTGG-3', exon 9, codons 338-343; and 5'-CATYTGYTCRTGDATCCAATC-3', exon 11, codons 442-448 of TMPRSS3). The full-length Tmprss 3 cDNA was isolated by $5^{\prime}$ and $3^{\prime}$ RACE. Tmprss 3 introns were amplified from BAC 556K 2 using primers based on predicted Tmprss 3 exons. Additional sequences were identified by BLASTN analyses of the Celera Mouse database (http:// www.celera.com/). Sequences were assembled using the GAP4 Staden software (35). Sequences of the primers used are listed in http://medgen.unige.ch/public_1.html

\section{Expression pattern of Tmprss3}

$R T-P C R . \quad$ RT-PCR with primers $5^{\prime}$-CCTGGAGTCTATACGCGAATCAC- $3^{\prime}$ and $5^{\prime}$-ATTGGGTGGAGATCTGAGATCTG-3' located within exons 11 and 12 of Tmprss3, respectively, was performed on a normalized cDNA panel as described (36). Intron 11 of Tmprss 3 (3198 bp) is sufficiently large such that amplification from genomic DNA should yield either no PCR product or an easily distinguishable product.

RT-PCR was performed on RNA extracted from 5-day-old rat modiolus, stria vascularis, organ of Corti and cultured spiral ganglia cells, as described (37). PCRs for rat Tmprss3, were performed using primers based on mouse nucleotide sequence. PCRs for rat $\mathrm{ENaC} \alpha$-, $\beta$-, and $\gamma$-subunits were performed using primers based on rat nucleotide sequences (GenBank Nos. NM_031548.1, X77932.1 and U37540.1, respectively). Sequences of the primers used are listed in http://medgen. unige.ch/public_1.html

RNA in situ hybridization. A $1.4 \mathrm{~kb}$ fragment from the $3^{\prime} \mathrm{UTR}$ of mouse Tmprss 3 was amplified using primers $5^{\prime}$-CTGAAGTGAACAAGCCTGGAGTC-3' and 5'-TAGTCATCTCTTACTGTGACTAG-3' and cloned in TOPO TA PCRII vector (Invitrogen, Paisley, UK). Digoxigenin-labelled sense and antisense riboprobes were synthesized using T7 and SP6 RNA polymerases, respectively. Heads of 5 day-old mice were fixed 

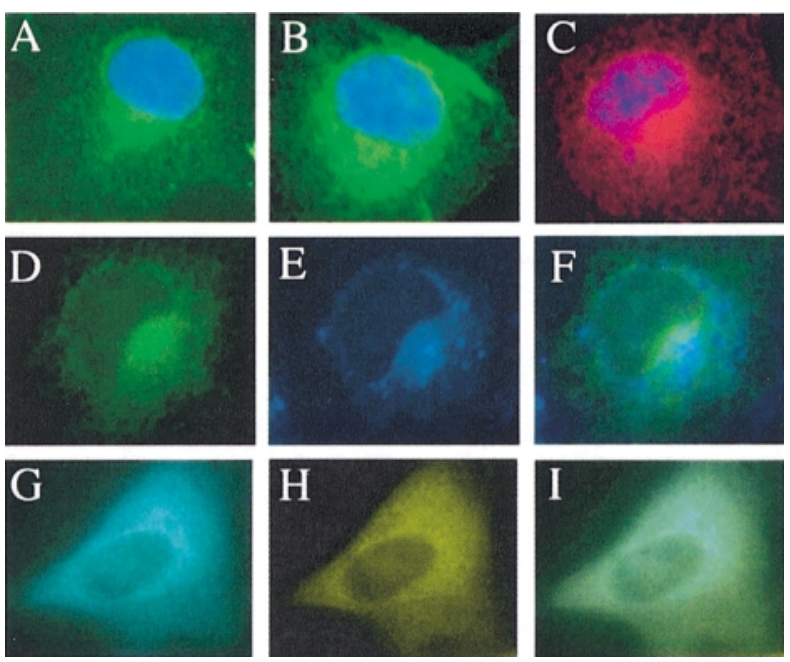

Figure 4. Intracellular localization of TMPRSS3 protein. Fluorescence microscopic analysis of fixed Cos-7 cells transiently transfected with pWT-TMPRSS3 (panel A, green signal), p5 $5^{\prime}$ EGFP-TMPRSS3 (panel $\mathbf{B}$, green signal) and p5 $5^{\prime}$ HA-TMPRSS3 (panel $\mathbf{C}$, red signal) and counterstained with 4,6-diamidoni2-phenylindole (DAPI) (panels A, B and C, blue signal). Fixed Cos-7 cells transiently transfected with pEGFP-TMPRSS3 (panel D, green signal) and counterstained with endoplasmic reticulum marker (ER-Tracker, panel E, blue signal). Co-localisation of EGFP-TMPRSS3 with ER marker is shown in panel F. Fixed HeLa cells transiently co-transfected with pTMPRSS3$3^{\prime}$ ECFP and pYFP-ER (panels G-I). TMPRSS3-ECFP signal is shown in panel G. pYFP signal is shown in panel H. Co-localisation of TMPRSS3-ECFP with the ER marker is shown in panel I.

in 4\% paraformaldehyde, washed in PBS, dehydrated and paraffin embedded. Sections, $8 \mu \mathrm{m}$ thick, were mounted onto superfrost slides. Slides were deparaffinated, rehydrated, acetylated $(0.25 \%$ acetic anhydride, $45 \mathrm{mM} \mathrm{NaCl}, 100 \mathrm{mM}$ Tris, $\mathrm{pH} 8)$ and washed in PBS. In situ hybridization was performed overnight at $58^{\circ} \mathrm{C}$ in $40 \%$ formamide, $5 \times \mathrm{SSC}, 1 \times$ Denhardt's, $100 \mu \mathrm{g} / \mathrm{ml}$ tRNA and the digoxigenin-labelled probe. After washing once with $2 \times \mathrm{SSC}$ at room temperature, twice with $2 \times \mathrm{SSC}$ and twice with $0.1 \times \mathrm{SSC}$ at $58^{\circ} \mathrm{C}$, sections were equilibrated with Triton washing buffer (TWB), $(0.05 \%$ Triton $\mathrm{X}-100,150 \mathrm{~mm} \mathrm{NaCl}, 100 \mathrm{~mm}$ Tris, $\mathrm{pH} 7.5)$. Non specific binding sites were blocked with TWB containing $0.5 \%$ blocking reagent (Roche, Rotkreuz, Switzerland) and sections were incubated with anti-digoxigenin antibody for $2 \mathrm{~h}$. After two washing steps with TWB and equilibration with alkaline phosphatase buffer NTMT $\left(100 \mathrm{mM} \mathrm{NaCl}, 50 \mathrm{mM} \mathrm{MgCl}_{2}, 100 \mathrm{mM}\right.$ Tris, $\mathrm{pH} 9.5,0.1 \%$ Tween 20), the slides were incubated with the substrate (NBT/BCIP) overnight at room temperature. The reaction was stopped in TE buffer ( $\mathrm{pH}$ 8.5). Sections were dehydrated and mounted. Images were recorded with a Hamamatsu digital camera.

cDNA constructs. To visualize the subcellular localization of TMPRSS3, four plasmids that express either tagged or wild-type TMPRSS3 were constructed: WT-TMPRSS3 was PCR-amplified and subcloned into vectors pECFP-N1 (Clontech, Palo Alto, California), pcDNA3X(+)MycEGFP, pcDNA3X $(+) H A$ and pcDNA3.1 (Invitrogen). For expression studies, WT-TMPRSS3 was subcloned into pSD5 expression

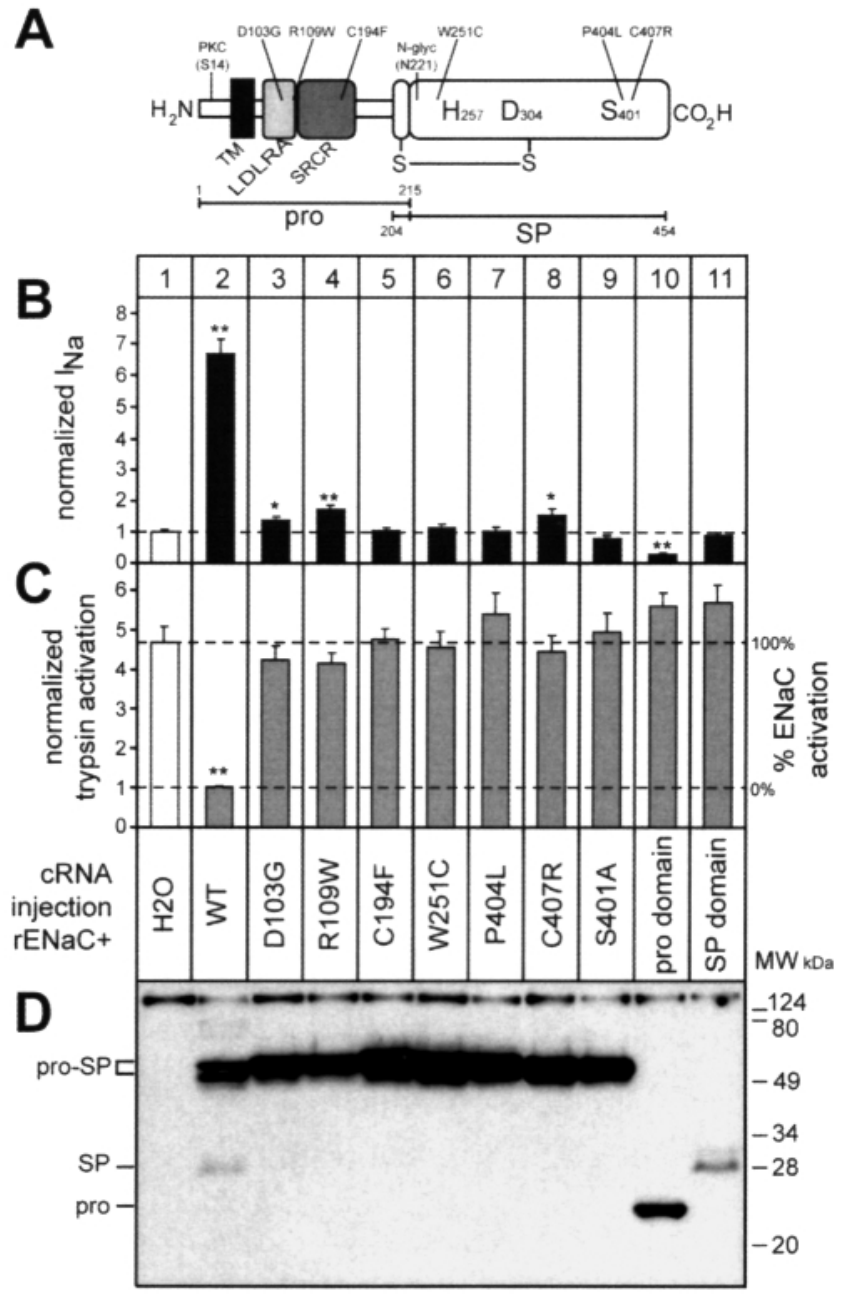

Figure 5. Functional expression of TMPRSS3 in Xenopus oocytes. (A) Predicted structure of TMPRSS3. Numbers indicate the amino acid positions. The serine protease catalytic triad residues (H257, D304 and S401), the predicted disulfide bridge which links the pro- and catalytic domains of TMPRSS3 and the tested human deafness associated TMPRSS3 missense mutations D103G, R109W, C194F, W251C, P404L and C407R are shown. TM, transmembrane domain; LDLRA, low-density lipoprotein receptor class A domain; SRCR; scavenger receptor cysteine-rich domain; pro, pro-domain; SP, serine protease domain. (B) Comparison of the effect of WT-TMPRSS3 and mutants on $\mathrm{I}_{\mathrm{Na}}$ in Xenopus oocytes. Oocytes were injected with rat $\mathrm{ENaC}$ subunits in the presence of either water (lane 1, white column), WT-TMPRSS3 (lane 2), TMPRSS3 missense deafness associated mutants (lanes 3-8), serine catalytic mutant (lane 9), pro domain construct (lane 10) or serine protease domain construct (SP) of TMPRSS3 (black columns). $* *=P<0.01 ; *=P<0.05$ versus 1 . Lanes $1-9$ and $11,(n \geq 25)$; lane 10 , $(n=16)$. (C) Measure of the fold increase of ENaC-mediated $\mathrm{I}_{\mathrm{Na}}$ in injected oocytes after $3 \mathrm{~min}$ of perfusion of trypsin. Same conditions as described under B. $* *=P<0.01$. Lanes $1-9$ and $11,(n \geq 16)$; lane $10,(n=10)$. (D) Biochemical analysis of WT and mutants TMPRSS3 by western blot on protein extracts from pools of 10 injected oocytes. Pro-SP, pro-serine protease; pro, pro-domain; SP, serine protease domain, Same conditions as described under B.

vector (9). Mutants (D103G, R109W, C194F, W251C, P404L, C407R, S401A) were generated using the QuickChange mutagenesis kit (Stratagene, La Jolla, CA, USA). Two truncated TMPRSS3 constructs (pro domain M1S215: TMPRSS3 without the catalytic domain and SP 
domain M-C204-T454: TMPRSS3 catalytic domain alone) were generated by PCR and subcloned into PSD5 vector (Fig. 5A). All constructs were verified by DNA sequencing. Sequences of the primers and restriction enzymes used are listed in http://medgen.unige.ch/public_1.html

Human TMPRSS3 polyclonal antibodies. Two polyclonal antibodies were raised in rabbits against a mixture of two synthetic peptides NH2-MGENDPPAVEAPFSFC-COOH (amino acids 1-16 of human TMPRSS3) and NH2-CFLDWIHEQMERDLKT-COOH (amino acids 439-454 of human TMPRSS3) (CovalAb, Lyon, France, http://www.covalab.com).

Subcellular localization of TMPRSS3 protein. Cos-7, U2OS and MDCK cells were transfected with $0.5 \mu \mathrm{g}$ of $5^{\prime}$ MycEGFP-TMPRSS3 3 or $5^{\prime}$ HA-TMPRSS3 3 or WT-TMPRSS3 using LipofectAMINE PLUS reagent (Life Technologies, Paisley, UK, \#10964-013) and fixed in 4\% paraformaldehyde. 5' MycEGFP-TMPRSS3 fluorescence was detected using epifluorescence microscopy (Zeiss Axiophot I, color Axiocam). 5' HA-TMPRSS3 was detected using anti-HA antibody (Santa Cruz Biotechnology, Santa Cruz, CA, USA, \#sc7392) followed by a rhodamine-conjugated secondary antibodies (Santa Cruz Biotechnology, \#sc-2093). WT-TMPRSS3 was detected using rabbit anti-TMPRSS3 serum (CovalAb) followed by fluorescein isothiocyanate-conjugated secondary antibodies (Santa Cruz Biotechnology, \#sc-2090). Images were processed with the Adobe Photoshop software.

To perform co-localization experiments, transfected cells were incubated with markers for the Golgi apparatus (mouse anti-Giantin antibody, Calbiochem, San Diego, CA, USA, \#324450) followed by rhodamine-conjugated secondary antibody (Santa Cruz \#sc-2093) or the endoplasmic reticulum (ER-Tracker Blue-White DPX, Molecular Probes, Eugene, OR, USA, \#E-2353). Alternatively, HeLa and Cos-7 cells were cotransfected with $1 \mu \mathrm{g}$ of pTMPRSS3-ECFP-N1 and marker vectors including pEYFP-Peroxi, pEYFP-Mito, pEYFP-Golgi, pEYFP-ER, or pEYFP-Mem (Clontech) using Fugene 6 (Roche). Empty expression vectors were used as negative controls.

Electrophysiological measurements in Xenopus oocytes. Expression studies were performed in stage V/VI oocytes isolated from Xenopus laevis (Noerdhoek, South Africa). Oocytes were injected with $0.25 \mathrm{ng}$ of each cRNA coding for the rat $\alpha$, $\beta$ - and $\gamma-\mathrm{ENaC}$ subunits in the presence or absence of $2.5 \mathrm{ng}$ of wild-type or mutant TMPRSS3 cRNA in a total volume of $100 \mathrm{nl}$. Oocytes were incubated in modified Barth saline (MBS) solution and $48 \mathrm{~h}$ after injection, electrophysiological measurements were performed. Using the two-electrode voltage clamp technique, the amiloride-sensitive current $\left(\mathrm{I}_{\mathrm{Na}}\right)$ was measured in the presence of $120 \mathrm{mM}$ of $\mathrm{Na}^{+}$in Frog Ringer with $5 \mu \mathrm{M}$ amiloride at a holding potential of $-100 \mathrm{mV}$. The oocytes were perfused with $2 \mu \mathrm{g} / \mathrm{ml}$ of trypsin during 2-3 min and $\mathrm{I}_{\mathrm{Na}}$ was re-measured. All results are reported as means \pm SEM. Comparing independent sets of data, unpaired $t$-tests were used to determine significance. In experiments where oocytes were perfused with trypsin, paired $t$-tests were used. $(n)$ Represents the number of experiments performed.

Western blot analysis. Injected oocytes were incubated in MBS for $48 \mathrm{~h}$. Microsomal membrane protein extracts were obtained from pools of 10 injected oocytes lysed as described (38). Proteins were separated on a $10 \%$ SDS-polyacrylamide gel under reducing and denaturing conditions and transferred to nitrocellulose membrane. The membrane was processed using rabbit anti-TMPRSS3 serum (Covalab) according to standard procedures.

\section{ACKNOWLEDGEMENTS}

The laboratory of S.E.A. is supported by grants from the Swiss FNRS, the OFES/EU, the Foundation Child Care and funds from the University and Cantonal Hospital of Geneva. The laboratory of N.S. is supported by a Grant-in-Aid for Scientific Research and Fund for "Research for the Future" Program from the Japan Society for the Promotion of Science (JSPS). The laboratory of H.S.S. is supported by a "the Nossal Leadership" award from the Walter and Eliza Hall Institute of Medical Research and the National Health and Medical Research Council of Australia (project grant 215305 and fellowship 171601). The laboratory of B.C.R. is supported by grants from SNF. M.G. is partially supported by a Research Training Fellowship from the Garnett Passe and Rodney Williams Memorial Foundation. We thank Pauline Crewther for preparation of the manuscript.

\section{REFERENCES}

1. Morton, N.E. (1991) Genetic epidemiology of hearing impairment. Ann. NY Acad. Sci., 630, 16-31.

2. Cohen, M.J. and Gorlin, R.J. (1995) Epidemiology, Aetiology and Genetics Patterns. Oxford University Press, Oxford, UK.

3. Scott, H.S., Kudoh, J., Wattenhofer, M., Shibuya, K., Berry, A., Chrast, R., Guipponi, M., Wang, J., Kawasaki, K., Asakawa, S. et al. (2001) Insertion of beta-satellite repeats identifies a transmembrane protease causing both congenital and childhood onset autosomal recessive deafness. Nat. Genet., 27, 59-63.

4. Ben-Yosef, T., Wattenhofer, M., Riazuddin, S., Ahmed, Z.M., Scott, H.S., Kudoh, J., Shibuya, K., Antonarakis, S.E., Bonne-Tamir, B., Radhakrishna, U. et al. (2001) Novel mutations of TMPRSS3 in four DFNB8/B10 families segregating congenital autosomal recessive deafness. J. Med. Genet., 38, 396-400.

5. Masmoudi, S., Antonarakis, S.E., Schwede, T., Ghorbel, A.M., Gratri, M., Pappasavas, M.P., Drira, M., Elgaied-Boulila, A., Wattenhofer, M., Rossier, C. et al. (2001) Novel missense mutations of TMPRSS3 in two consanguineous Tunisian families with non-syndromic autosomal recessive deafness. Hum. Mutat., 18, 101-108.

6. Wattenhofer, M., Di Iorio, V., Rabionet, R., Dougherty, L., Pampanos, A., Schwede, T., Montserrat-Sentis, B., Arbones, L., Iliades, T., Pasquadibisceglie, A. et al. (2002) Mutations in the TMPRSS3 gene are a rare cause of childhood nonsyndromic deafness in Caucasian patients. J. Mol. Med., 80, 124-131.

7. Hooper, J.D., Clements, J.A., Quigley, J.P. and Antalis, T.M. (2001) Type II transmembrane serine proteases. Insights into an emerging class of cell surface proteolytic enzymes. J. Biol. Chem., 276, 857-860.

8. Vallet, V., Chraibi, A., Gaeggeler, H.P., Horisberger, J.D. and Rossier, B.C (1997) An epithelial serine protease activates the amiloride-sensitive sodium channel. Nature, 389, 607-610.

9. Vuagniaux, G., Vallet, V., Jaeger, N.F., Pfister, C., Bens, M., Farman, N., Courtois-Coutry, N., Vandewalle, A., Rossier, B.C. and Hummler, E. (2000) 
Activation of the amiloride-sensitive epithelial sodium channel by the serine protease mCAP1 expressed in a mouse cortical collecting duct cell line. J. Am. Soc. Nephrol., 11, 828-834.

10. Vuagniaux, G., Vallet, V., Jaeger, N.F., Hummler, E. and Rossier, B.C. (2002) Synergistic activation of $\mathrm{ENaC}$ by three membrane-bound channel-activating serine proteases (mCAP1, mCAP2, and mCAP3) and serum- and glucocorticoid-regulated kinase (Sgk 1) in Xenopus oocytes. J. Gen. Physiol., 120, 191-201.

11. Grunder, S., Muller, A. and Ruppersberg, J.P. (2001) Developmental and cellular expression pattern of epithelial sodium channel alpha, beta and gamma subunits in the inner ear of the rat. Eur. J. Neurosci., 13, 641-648.

12. Couloigner, V., Fay, M., Djelidi, S., Farman, N., Escoubet, B., Runembert, I., Sterkers, O., Friedlander, G. and Ferrary, E. (2001) Location and function of the epithelial Na channel in the cochlea. Am. J. Physiol. Renal. Physiol., 280, F214-222.

13. Steel, K.P. and Kros, C.J. (2001) A genetic approach to understanding auditory function. Nat. Genet., 27, 143-149.

14. Resendes, B.L., Williamson, R.E. and Morton, C.C. (2001) At the speed of sound: gene discovery in the auditory system. Am. J. Hum. Genet., 69 , 923-935.

15. Yamasaki, M., Komune, S., Shimozono, M., Matsuda, K. and Haruta, A. (2000) Development of monovalent ions in the endolymph in mouse cochlea. ORL J. Otorhinolaryngol. Relat. Spec., 62, 241-246.

16. Hanwell, D., Ishikawa, T., Saleki, R. and Rotin, D. (2002) Trafficking and cell surface stability of the epithelial $\mathrm{Na}^{+}$channel expressed in epithelial Madin-Darby canine kidney cells. J. Biol. Chem., 277, 9772-9779.

17. Iwasa, K.H., Mizuta, K., Lim, D.J., Benos, D.J. and Tachibana, M. (1994) Amiloride-sensitive channels in marginal cells in the stria vascularis of the guinea pig cochlea. Neurosci. Lett., 172, $163-166$.

18. Tu, T.Y., Chiu, J.H., Yang, W.K., Chang, T.J., Yang, A.H., Shu, C.H. and Lien, C.F. (1998) Establishment and characterization of a strial marginal cell line maintaining vectorial electrolyte transport. Hear Res., 123, 97-110.

19. Marcus, D.C. and Chiba, T. (1999) $\mathrm{K}^{+}$and $\mathrm{Na}^{+}$absorption by outer sulcus epithelial cells. Hear Res., 134, 48-56.

20. Chiba, T. and Marcus, D.C. (2000) Nonselective cation and BK channels in apical membrane of outer sulcus epithelial cells. J. Membr. Biol., 174, $167-179$.

21. Chiba, T. and Marcus, D.C. (2001) Basolateral $\mathrm{K}^{+}$conductance establishes driving force for cation absorption by outer sulcus epithelial cells. J. Membr. Biol., 184, 101-112.

22. Canessa, C.M., Schild, L., Buell, G., Thorens, B., Gautschi, I., Horisberger, J.D. and Rossier, B.C. (1994) Amiloride-sensitive epithelial $\mathrm{Na}^{+}$channel is made of three homologous subunits. Nature, 367, 463-467.

23. Firsov, D., Schild, L., Gautschi, I., Merillat, A.M., Schneeberger, E. and Rossier, B.C. (1996) Cell surface expression of the epithelial Na channel and a mutant causing Liddle syndrome: a quantitative approach. Proc. Natl Acad. Sci. USA, 93, 15370-15371.
24. Hummler, E., Barker, P., Gatzy, J., Beermann, F., Verdumo, C., Schmidt, A., Boucher, R. and Rossier, B.C. (1996) Early death due to defective neonatal lung liquid clearance in alpha-ENaC-deficient mice. Nat. Genet., 12, 325-328.

25. Barker, P.M., Nguyen, M.S., Gatzy, J.T., Grubb, B., Norman, H., Hummler, E., Rossier, B., Boucher, R.C. and Koller, B. (1998) Role of gammaENaC subunit in lung liquid clearance and electrolyte balance in newborn mice. Insights into perinatal adaptation and pseudohypoaldosteronism. J. Clin. Invest., 102, 1634-1640.

26. McDonald, F.J., Yang, B., Hrstka, R.F., Drummond, H.A., Tarr, D.E., McCray, P.B., Jr., Stokes J.B., Welsh, M.J. and Williamson, R.A. (1999) Disruption of the beta subunit of the epithelial $\mathrm{Na}^{+}$channel in mice: hyperkalemia and neonatal death associated with a pseudohypoaldosteronism phenotype. Proc. Natl Acad. Sci. USA, 96, 1727-1731.

27. Rusch, A. and Hummler, E. (1999) Mechano-electrical transduction in mice lacking the alpha-subunit of the epithelial sodium channel. Hear Res., 131, 170-176.

28. Oh, Y.S. and Warnock, D.G. (2000) Disorders of the epithelial $\mathrm{Na}(+)$ channel in Liddle's syndrome and autosomal recessive pseudohypoaldosteronism type 1. Exp. Nephrol., 8, 320-325.

29. Fritzsch, B., Pirvola, U. and Ylikoski, J. (1999) Making and breaking the innervation of the ear: neurotrophic support during ear development and its clinical implications. Cell Tissue Res., 295, 369-382.

30. Lee, R., Kermani, P., Teng, K.K. and Hempstead, B.L. (2001) Regulation of cell survival by secreted proneurotrophins. Science, 294, 1945-1948.

31. Gestwa, G., Wiechers, B., Zimmermann, U., Praetorius, M., Rohbock, K., Kopschall, I., Zenner, H.P. and Knipper, M. (1999) Differential expression of trkB.T1 and trkB.T2, truncated trkC, and p75(NGFR) in the cochlea prior to hearing function. J. Comp. Neurol., 414, 33-49.

32. Wiechers, B., Gestwa, G., Mack, A., Carroll, P., Zenner, H.P. and Knipper, M. (1999) A changing pattern of brain-derived neurotrophic factor expression correlates with the rearrangement of fibers during cochlear development of rats and mice. J. Neurosci., 19, 3033-3042.

33. Farinas, I., Jones, K.R., Tessarollo, L., Vigers, A.J., Huang, E., Kirstein, M., de Caprona, D.C., Coppola, V., Backus, C., Reichardt, L.F. and Fritzsch, B. (2001) Spatial shaping of cochlear innervation by temporally regulated neurotrophin expression. J. Neurosci., 21, 6170-6180.

34. Samant, S.A., Fossella, J., Silver, L.M. and Pilder, S.H. (1999) Mapping and cloning recombinant breakpoints demarcating the hybrid sterility 6-specific sperm tail assembly defect. Mamm. Genome, 10, 88-94.

35. Staden, R. (1996) The Staden sequence analysis package. Mol. Biotechnol., 5, 233-241.

36. Michaud, J., Kudoh, J., Berry, A., Bonne-Tamir, B., Lalioti, M.D., Rossier, C., Shibuya, K., Kawasaki, K., Asakawa, S., Minoshima, S. et al. (2000) Isolation and characterization of a human chromosome 21q22.3 gene (WDR4) and its mouse homologue that code for a WD-repeat protein. Genomics, 68, 71-79.

37. Marzella, P.L., Clark, G.M., Shepherd, R.K., Bartlett, P.F. and Kilpatrick, T.J. (1997) LIF potentiates the NT-3-mediated survival of spiral ganglia neurones in vitro. Neuroreport, 8, 1641-1644.

38. Geering, K., Theulaz, I., Verrey, F., Hauptle, M.T. and Rossier, B.C. (1989) A role for the beta-subunit in the expression of functional $\mathrm{Na}^{+}-\mathrm{K}^{+}$-ATPase in Xenopus oocytes. Am. J. Physiol., 257, C851-858. 